CRYSTALLOGRAPHIC COMMUNICATIONS

ISSN 2056-9890

Received 22 November 2018

Accepted 10 December 2018

Edited by H. Ishida, Okayama University, Japan

‡ Thomson Reuters ResearcherID: F-88162012.

$\S$ Thomson Reuters ResearcherID: A-55252009.

Keywords: crystal structure; chalcone; hydrogen bond; Hirshfeld surface analysis.

CCDC reference: 1036743

Supporting information: this article has supporting information at journals.iucr.org/e

\section{Crystal structure and Hirshfeld surface analysis of a chalcone derivative: $(E)-3-(4-$ fluorophenyl)-1-(4- nitrophenyl)prop-2-en-1-one}

\author{
Qin Ai Wong, ${ }^{\text {a }}$ Tze Shyang Chia, ${ }^{a} \neq$ Huey Chong Kwong, ${ }^{\text {b }}$ C. S. Chidan Kumar, ${ }^{\mathrm{c} *}$ \\ Ching Kheng Quah ${ }^{\mathrm{a}} \S$ and Md. Azharul Arafath ${ }^{\mathrm{d}}$ *
}

aX-ray Crystallography Unit, School of Physics, Universiti Sains Malaysia, 11800 USM, Penang, Malaysia, 'bSchool of
Chemical Sciences, Universiti Sains Malaysia, 11800 USM, Penang, Malaysia, 'Department of Engineering Chemistry,
Vidya Vikas Institute of Engineering and Technology, Visvesvaraya Technological University, Alanahalli, Mysuru 570
028, India, and 'Department of Chemistry, Shahjalal University of Science and Technology, Sylhet, 3114, Bangladesh.
${ }^{*}$ Correspondence e-mail: chidankumar@gmail.com, arafath_sustche90@yahoo.com

The molecular structure of the title chalcone derivative, $\mathrm{C}_{15} \mathrm{H}_{10} \mathrm{FNO}_{3}$, is nearly planar and the molecule adopts a trans configuration with respect to the $\mathrm{C}=\mathrm{C}$ double bond. The nitro group is nearly coplanar with the attached benzene ring, which is nearly parallel to the second benzene ring. In the crystal, molecules are connected by pairs of weak intermolecular $\mathrm{C}-\mathrm{H} \cdots \mathrm{O}$ hydrogen bonds into inversion dimers. The dimers are further linked by another $\mathrm{C}-\mathrm{H} \cdots \mathrm{O}$ hydrogen bond and a $\mathrm{C}-\mathrm{H} \cdots \mathrm{F}$ hydrogen bond into sheets parallel to (104). $\pi-\pi$ interactions occur between the sheets, with a centroid-centroid distance of 3.8860 (11) ̊.. Hirshfeld surface analysis was used to investigate and quantify the intermolecular interactions.

\section{Chemical context}

Non-linear optics (NLO) is the study of interactions between intense light and matter, in which the dielectric polarization responds non-linearly to the electric field of the light. This non-linearity leads to frequency-mixing processes (second-, third- and high-harmonic generations), the optical Kerr effect etc (Boulanger \& Zyss, 2006). Chalcone is one of the NLO materials and is known for its high NLO coefficients and good crystallizability (Prabhu et al., 2013). Donor-acceptor substituted chalcone derivatives consist of two substituted phenyl rings covalently bonded to the ends of a $\alpha, \beta$-unsaturated propenone bridge $(\mathrm{C}=\mathrm{C}-\mathrm{C}=\mathrm{O})$, which provides the necessary configuration for intramolecular charge transfer to show NLO properties (Fun et al., 2011). However, organic chalcone derivatives with a low melting point are at a disadvantage for applications as optical instruments. In a continuation of our ongoing studies on non-linear optical properties of various chalcone derivatives (Chandra Shekhara Shetty et al., 2017; Ekbote et al., 2017; Kwong et al., 2018), we report herein the synthesis, structure determination and Hirshfeld surface analysis of the title compound.<smiles>O=C(/C=C/c1ccc(F)cc1)c1ccc([N+](=O)[O-])cc1</smiles> 


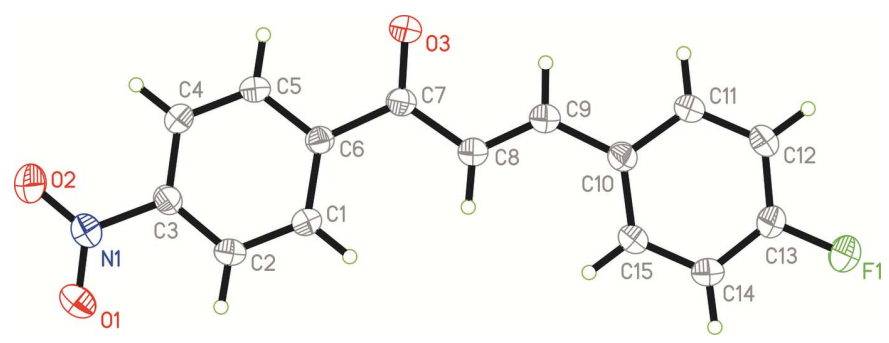

Figure 1

The molecular structure of the title compound with atom labels and $30 \%$ probability displacement ellipsoids.

\section{Structural commentary}

The asymmetric unit of the title chalcone derivative consists of a unique molecule, containing two para-substituted phenyl rings and an enone connecting bridge (Fig. 1). The molecule adopts a trans configuration with respect to the $\mathrm{C} 8=\mathrm{C} 9$ olefinic double bond, as indicated by the $\mathrm{C} 7-\mathrm{C} 8-\mathrm{C} 9-\mathrm{C} 10$ torsion angle of $-179.96(15)^{\circ}$. The $\mathrm{C} 7=\mathrm{O} 3$ carbonyl group adopts an $s$-cis configuration with respect to the $\mathrm{C} 8=\mathrm{C} 9$ double bond as indicated by $\mathrm{O} 3-\mathrm{C} 7-\mathrm{C} 8-\mathrm{C} 9$ torsion angle of $-0.8(3)^{\circ}$. The molecule (excluding $\mathrm{H}$ atoms) is nearly planar with a maximum deviation of 0.103 (2) $\AA$ at atom O1 of the terminal nitro group. The nitro group is nearly coplanar with the attached $\mathrm{C} 1-\mathrm{C} 6$ benzene ring as indicated by the small dihedral angle of $7.9(2)^{\circ}$. The C1-C6 and C10-C15 benzene rings make a small dihedral angle of $4.27(8)^{\circ}$ with each other.

\section{Supramolecular features}

In the crystal, molecules are connected by pairs of weak $\mathrm{C}-$ $\mathrm{H} \cdot \mathrm{O} \mathrm{O}$ hydrogen bonds $\left(\mathrm{C} 11-\mathrm{H} 11 A \cdots \mathrm{O} 3^{\text {ii }}\right.$; symmetry code as in Table 1) into inversion dimers with an $R_{2}^{2}(14)$ ring motif. These dimers are further linked by $\mathrm{C}-\mathrm{H} \cdots \mathrm{O}$ and $\mathrm{C}-\mathrm{H} \cdots \mathrm{F}$ hydrogen bonds $\left(\mathrm{C} 15-\mathrm{H} 15 A \cdots \mathrm{O} 1^{\mathrm{iii}}\right.$ and $\mathrm{C} 4-\mathrm{H} 4 A \cdots \mathrm{F} 1^{\mathrm{i}}$; Table 1) into two-dimensional sheets parallel to (104) (Fig. 2).

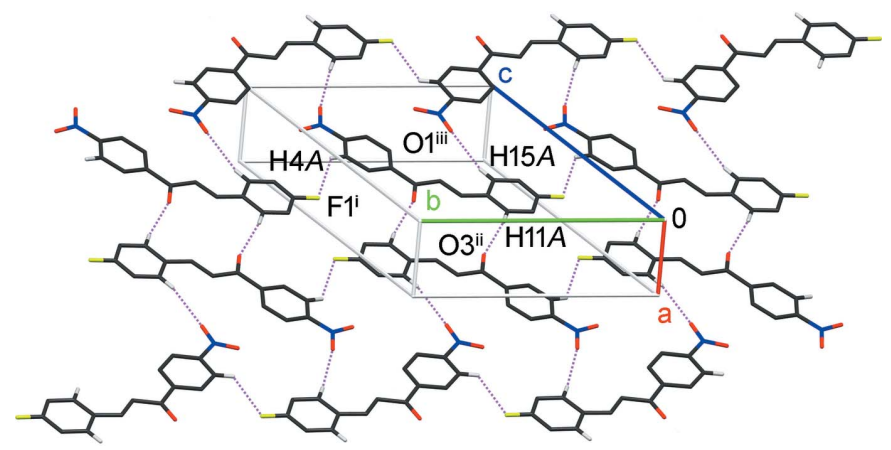

Figure 2

A partial packing diagram of the title compound, showing a twodimensional sheet formed by $\mathrm{C}-\mathrm{H} \cdots \mathrm{O}$ and $\mathrm{C}-\mathrm{H} \cdots \mathrm{F}$ hydrogen bonds (dotted lines). $\mathrm{H}$ atoms not involved in hydrogen bonding are omitted for clarity. [Symmetry codes: (i) $x, y+1, z$; (ii) $-x+2,-y+1,-z+1$; (iii) $-x$, $y-\frac{1}{2},-z+\frac{3}{2}$.]
Table 1

Hydrogen-bond geometry $\left(\AA,^{\circ}\right)$.

\begin{tabular}{lllll}
\hline$D-\mathrm{H} \cdots A$ & $D-\mathrm{H}$ & $\mathrm{H} \cdots A$ & $D \cdots A$ & $D-\mathrm{H} \cdots A$ \\
\hline $\mathrm{C} 4-\mathrm{H} 4 A \cdots \mathrm{F} 1^{\mathrm{i}}$ & 0.93 & 2.53 & $3.183(2)$ & 128 \\
$\mathrm{C} 11-\mathrm{H} 11 A \cdots \mathrm{O} 3^{\mathrm{ii}}$ & 0.93 & 2.43 & $3.329(2)$ & 161 \\
$\mathrm{C} 15-\mathrm{H} 15 A \cdots \mathrm{O} 1^{\mathrm{iii}}$ & 0.93 & 2.58 & $3.489(2)$ & 166 \\
\hline
\end{tabular}

Symmetry codes: (i) $x, y+1, z$; (ii) $-x+2,-y+1,-z+1$; (iii) $-x, y-\frac{1}{2},-z+\frac{3}{2}$.

Weak $\pi-\pi$ interactions occur between the sheets $\left[C g 1 \cdots C g 1^{\mathrm{iv}, \mathrm{v}}\right.$ and $C g 2 \cdots C g 2^{\mathrm{iv}, \mathrm{v}}=3.8860(11) \AA$, where $C g 1$ and $C g 2$ are the centroids of $\mathrm{C} 1-\mathrm{C} 6$ and $\mathrm{C} 10-\mathrm{C} 15$ benzene rings, respectively; symmetry codes: (iv) $x-1, y, z$; (v) $x+1, y$, z] (Fig. 3).

\section{Hirshfeld surface analysis}

The Hirsheld surfaces mapped with normalized contact distance $d_{\text {norm }}$ and electrostatic potentials, and the twodimensional fingerprint plot were generated using CrystalExplorer (Version 17.5; Spackman \& Jayatilaka, 2009; Spackman \& McKinnon, 2002; Spackman et al., 2008; Turner et al., 2017). The darkest red spots on the Hirshfeld surface mapped with $d_{\text {norm }}$ [Fig. 4(a)] correspond to the C11$\mathrm{H} 11 A \cdots \mathrm{O} 3$ hydrogen bond. The $\mathrm{C} 4-\mathrm{H} 4 A \cdots \mathrm{F} 1$ and $\mathrm{C} 15-$ $\mathrm{H} 15 A \cdots \mathrm{O} 1$ hydrogen bonds are indicated as two pairs of lighter red spots on the $d_{\text {norm }}$ surface. The H12A ․ F1 contact, with its $\mathrm{H}$..F distance shorter than the sum of van der Waals radii by $0.01 \AA$, appears as two tiny red spots on the $d_{\text {norm }}$ surface. The donor and acceptor of a hydrogen bond with positive and negative electrostatic potentials, respectively, are

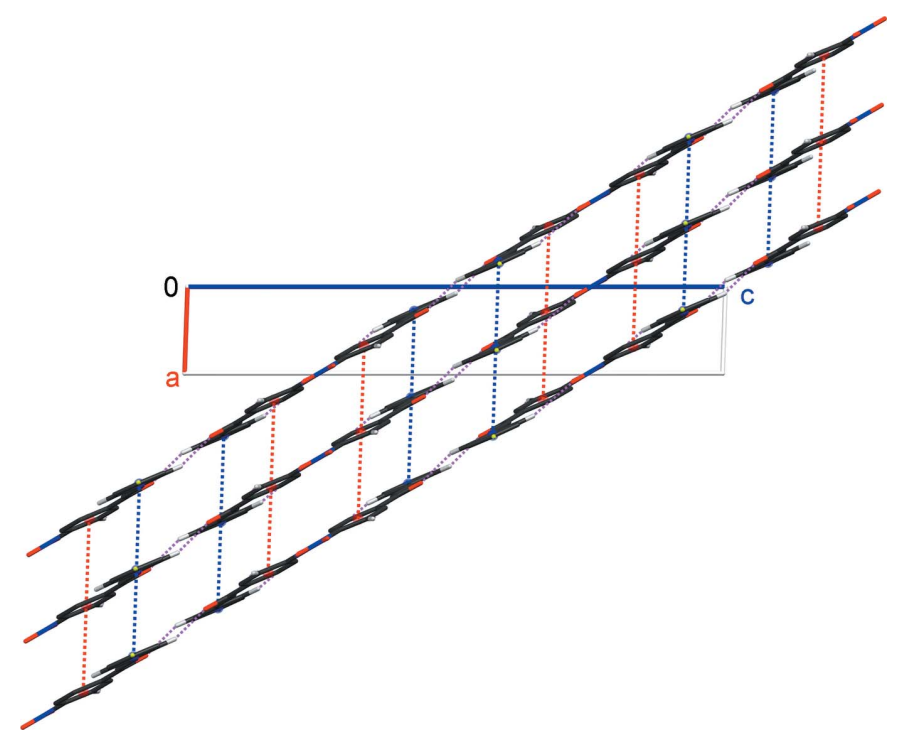

Figure 3

A partial packing diagram of the title compound, showing three separated sheets parallel to (104). The intermolecular $\pi-\pi$ interactions between adjacent sheets are represented as red and blue dashed lines, involving $C g 1 \cdots C g 1$ and $C g 2 \cdots C g 2$, respectively. $C g 1$ and $C g 2$ are the centroids of the $\mathrm{C} 1-\mathrm{C} 6$ and $\mathrm{C} 10-\mathrm{C} 15$ benzene rings, respectively. 

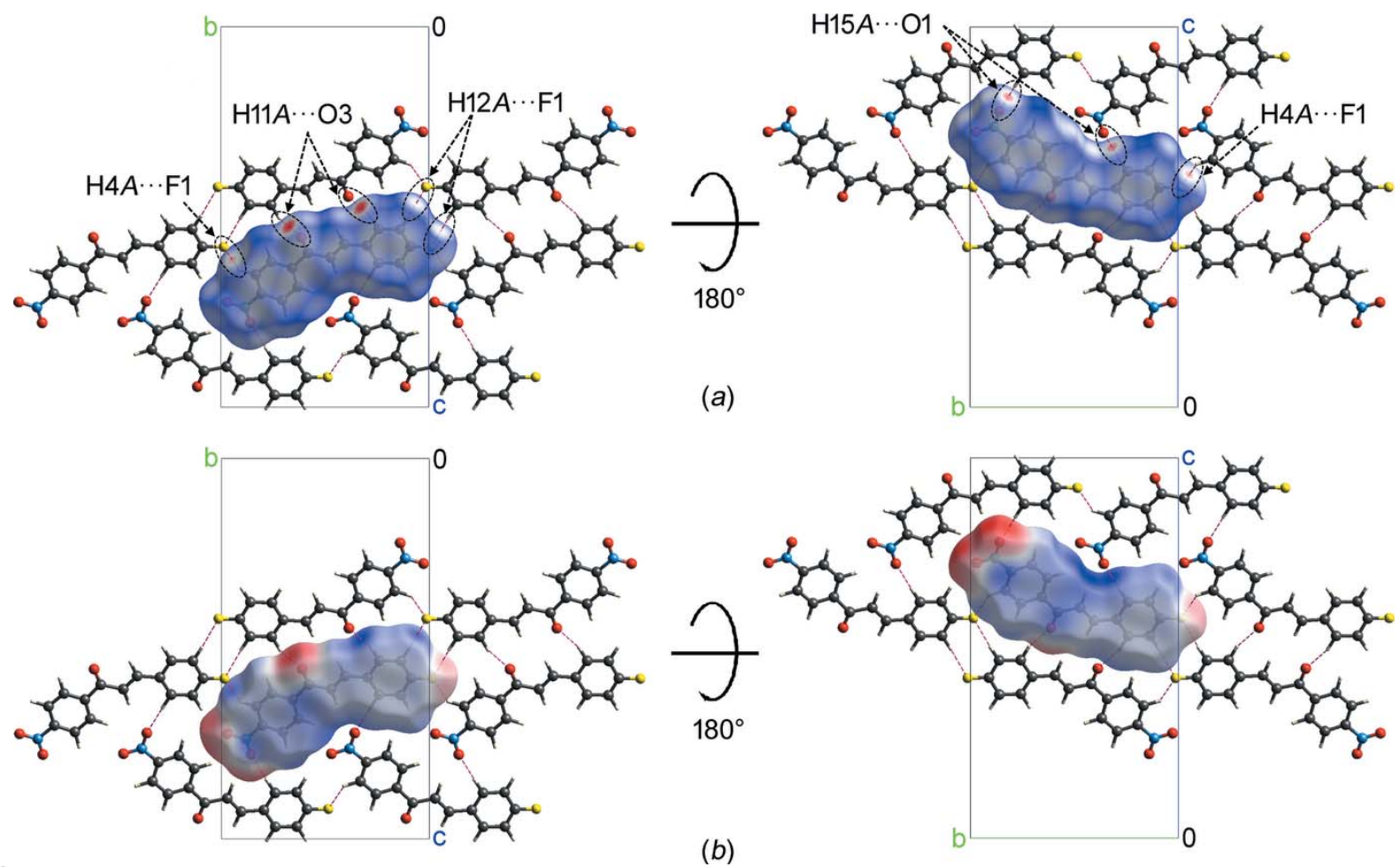

Figure 4

The Hirshfeld surfaces mapped with $(a) d_{\text {norm }}$ and $(b)$ electrostatic potential for the central molecule of the title compound surrounded by six neighbouring molecules.
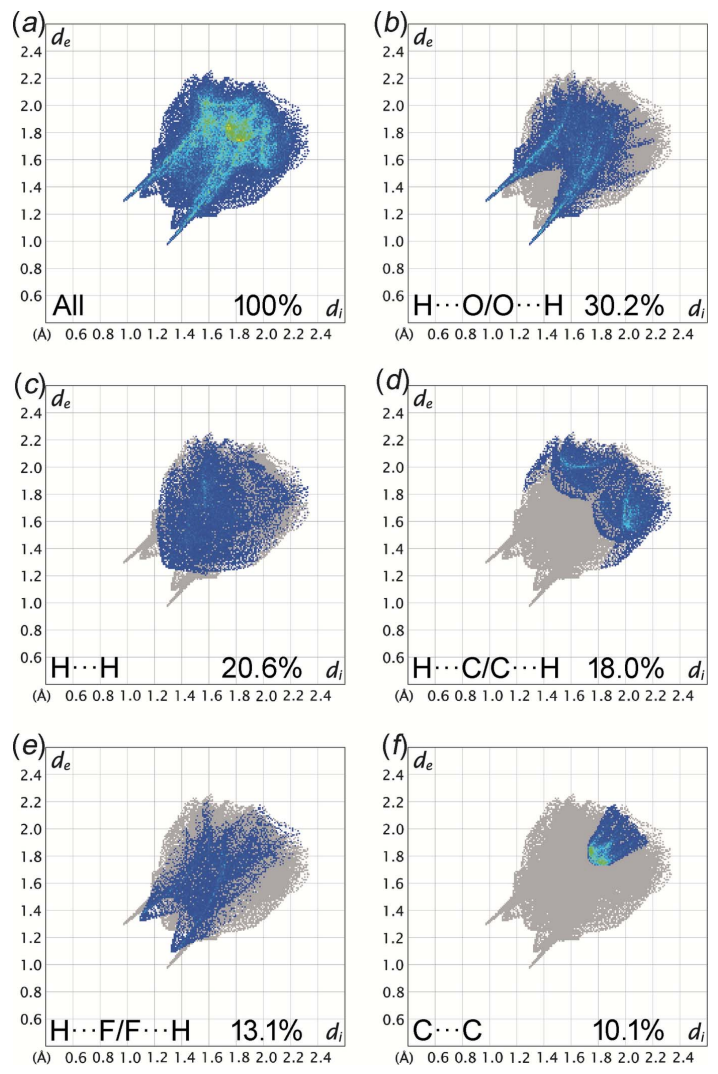

Figure 5

The two-dimensional fingerprint plots of the title compound for different intermolecular contacts and their percentage contributions to the Hirshfeld surface. $d_{\mathrm{i}}$ and $d_{\mathrm{e}}$ are the distances from the Hirshfeld surface to the nearest atom interior and exterior, respectively, to the surface. represented as blue and red regions on the Hirshfeld surface mapped with electrostatic potential [Fig. 4(b)]. The electrostatic potential of the $\mathrm{F}$ atom is less negative as compared to the $\mathrm{O}$ atoms of nitro and carbonyl groups, as indicated by the lighter red region. The $\mathrm{H} \cdots \mathrm{O} / \mathrm{O} \cdots \mathrm{H}$ contacts are the most populated contacts and contribute $30.2 \%$ of the total intermolecular contacts, followed by $\mathrm{H} \cdots \mathrm{H}(20.6 \%), \mathrm{H} \cdots \mathrm{C} / \mathrm{C} \cdots \mathrm{H}$ $(18.0 \%), \mathrm{H} \cdots \mathrm{F} / \mathrm{F} \cdots \mathrm{H}(13.1 \%)$ and $\mathrm{C} \cdot \mathrm{C}(10.1 \%)$ contacts (Fig. 5). The shortest $\mathrm{H} \cdots \mathrm{O} / \mathrm{O} \cdots \mathrm{H}$ and $\mathrm{H} \cdots \mathrm{F} / \mathrm{F} \cdots \mathrm{H}$ contacts are represented as the tips of the pseudo-mirrored sharp spikes and blunt peaks at $d_{\mathrm{e}}+d_{\mathrm{i}} \simeq 2.3$ and $2.4 \AA$, respectively, which correspond to the $\mathrm{C} 11-\mathrm{H} 11 A \cdots \mathrm{O} 3$ and $\mathrm{C} 4-$ $\mathrm{H} 4 A \cdots \mathrm{F} 1 \mathrm{hydrogen}$ bonds. The characteristic 'wings' are missing in the fingerprint plot of $\mathrm{H} \cdots \mathrm{C} / \mathrm{C} \cdots \mathrm{H}$ contacts, indicating the absence of any significant $\mathrm{C}-\mathrm{H} \cdots \pi$ interactions in the crystal. The C ..C contacts, including the intermolecular $\pi-\pi$ interactions, appear as a unique 'triangle' focused at $d_{\mathrm{e}} \simeq$ $d_{\mathrm{i}} \simeq 1.8 \AA$. The presence of significant $\pi-\pi$ interactions is supported by the unique pattern of red and blue 'triangles' on the shape-index surface (Fig. 6), and the flat regions on the curvedness surface (Fig. 7) of the benzene rings.

\section{Database survey}

The bond lengths and bond angles of the title compound are comparable with those in two similar structures, viz., (E)-1-(4nitrophenyl)-3-phenylprop-2-en-1-one (refcode BUDXOO; Jing, 2009a) and (E)-3-(4-fluorophenyl)-1-phenylprop-2-en-1one (refcode BUDYOP; Jing, 2009b) found in the Cambridge 


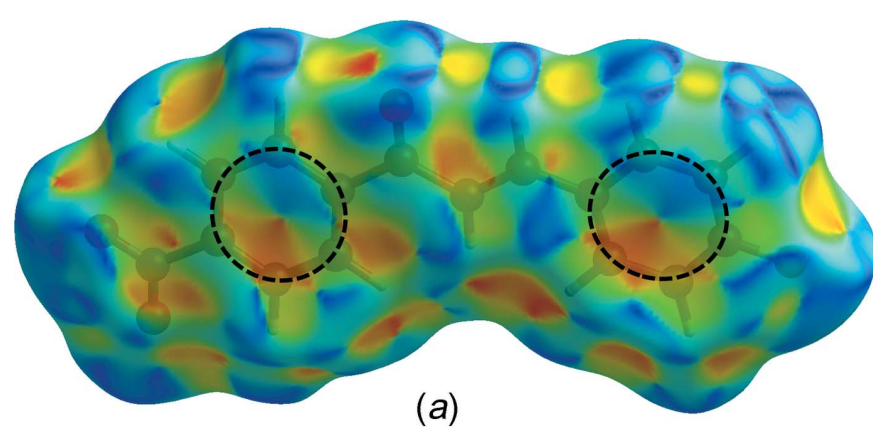

(a)

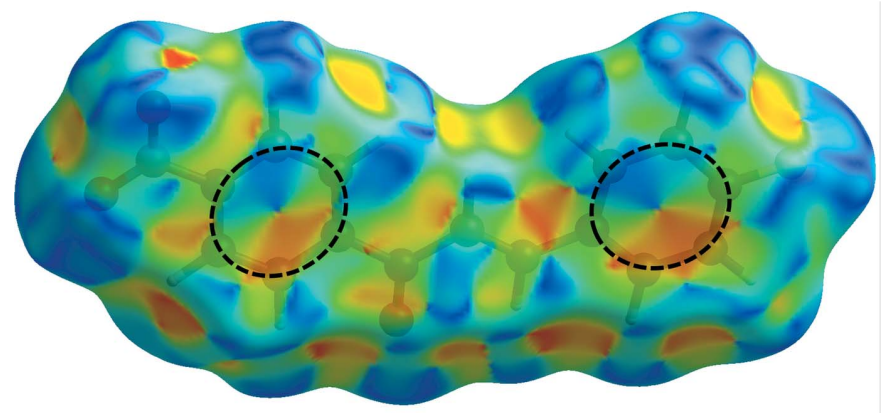

(b)

Figure 6

(a) Front and (b) rear views of the Hirshfeld surface mapped over shapeindex for the title compound. The dashed-line circles highlight unique patterns of red and blue 'triangles'.

Structural Database (Version 5.39; Groom et al., 2016). The molecular conformations of these two structures are nearly planar, with small dihedral angles of $5.00(6)$ and $10.60(11)^{\circ}$, respectively, between the phenyl rings.
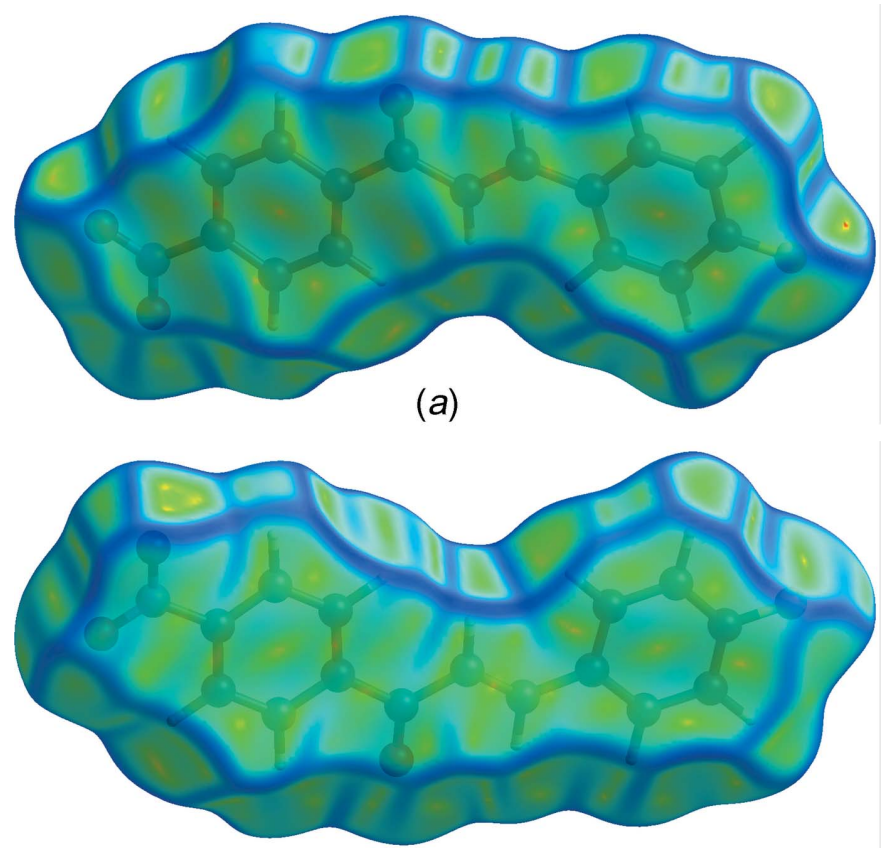

(b)

Figure 7

(a) Front and (b) rear views of the Hirshfeld surface mapped over curvedness.
Table 2

Experimental details.

Crystal data

Chemical formula

$M_{\mathrm{r}}$

Crystal system, space group

Temperature (K)

$a, b, c(\AA)$

$\beta\left({ }^{\circ}\right)$

$V\left(\AA^{3}\right)$

$Z$

Radiation type

$\mu\left(\mathrm{mm}^{-1}\right)$

Crystal size (mm)

$\mathrm{C}_{15} \mathrm{H}_{10} \mathrm{FNO}_{3}$

271.24

Monoclinic, $P 2_{1} / c$

296

3.8860 (5), 13.2324 (16), 24.199 (3)

$91.963(2)$

$1243.6(3)$

Mo $K \alpha$

0.11

$0.49 \times 0.35 \times 0.31$

Data collection

Diffractometer

Absorption correction

$T_{\min }, T_{\max }$

No. of measured, independent and observed $[I>2 \sigma(I)]$ reflections

$R_{\text {int }}$

$(\sin \theta / \lambda)_{\max }\left(\AA^{-1}\right)$

Bruker SMART APEXII DUO

CCD area-detector

Multi-scan ( $S A D A B S$; Bruker, 2009)

$0.794,0.926$

10823, 2418, 1922

0.026

0.617

Refinement

$R\left[F^{2}>2 \sigma\left(F^{2}\right)\right], w R\left(F^{2}\right), S$

No. of reflections

No. of parameters

$\mathrm{H}$-atom treatment

$\Delta \rho_{\max }, \Delta \rho_{\min }\left(\mathrm{e} \AA^{-3}\right)$

$0.043,0.138,1.04$

2418

181

$\mathrm{H}$-atom parameters constrained $0.21,-0.17$

Computer programs: APEX2 and SAINT (Bruker, 2009), SHELXS2013 and SHELXTL (Sheldrick, 2008), SHELXL2014 (Sheldrick, 2015), Mercury (Macrae et al., 2008), PLATON (Spek, 2009) and publCIF (Westrip, 2010).

\section{Synthesis and crystallization}

4-Nitroacetophenone $(1.65 \mathrm{~g}, \quad 0.01 \mathrm{~mol})$ and 4-fluorobenzaldehyde $(1.24 \mathrm{~g}, 0.01 \mathrm{~mol})$ were dissolved in methanol $(20 \mathrm{ml})$. A catalytic amount of $\mathrm{NaOH}$ was added to the solution dropwise with vigorous stirring. The reaction mixture was stirred for about $6 \mathrm{~h}$ at room temperature. The progress of the reaction was monitored by TLC. The formed crude product was filtered, washed repeatedly with distilled water and recrystallized from ethanol to obtain the title chalcone derivative. Yellowish single-crystals suitable for X-ray diffraction were obtained from an acetone solution by slow evaporation at room temperature.

\section{Refinement}

Crystal data, data collection and structure refinement details are summarized in Table 2. All $\mathrm{H}$ atoms were positioned geometrically $(\mathrm{C}-\mathrm{H}=0.93 \AA)$ and refined using a riding model with $U_{\text {iso }}(\mathrm{H})=1.2 U_{\text {eq }}(\mathrm{C})$.

\section{Funding information}

QAW thanks the Malaysian Government and USM for the award of the post of Research Officer under the Research University Individual Grant (1001/PFIZIK/8011080). HCK thanks the Malaysian Government for a MyBrain15 scholarship. 


\section{References}

Boulanger, B. \& Zyss, J. (2006). International Tables for Crystallography, Vol. D, ch. 1.7, 178-219.

Bruker (2009). APEX2, SAINT and SADABS. Bruker AXS Inc., Madison, Wisconsin, USA.

Chandra Shekhara Shetty, T., Chidan Kumar, C. S., Gagan Patel, K. N., Chia, T. S., Dharmaprakash, S. M., Ramasami, P., Umar, Y., Chandraju, S. \& Quah, C. K. (2017). J. Mol. Struct. 1143, 306-317.

Ekbote, A., Patil, P. S., Maidur, S. R., Chia, T. S. \& Quah, C. K. (2017). Dyes Pigments, 139, 720-729.

Fun, H.-K., Loh, W.-S., Sarojini, B. K., Khaleel, V. M. \& Narayana, B. (2011). Acta Cryst. E67, o2651-o2652.

Groom, C. R., Bruno, I. J., Lightfoot, M. P. \& Ward, S. C. (2016). Acta Cryst. B72, 171-179.

Jing, L.-H. (2009a). Acta Cryst. E65, o2510.

Jing, L.-H. (2009b). Acta Cryst. E65, o2515.

Kwong, H. C., Rakesh, M. S., Chidan Kumar, C. S., Maidur, S. R., Patil, P. S., Quah, C. K., Win, Y.-F., Parlak, C. \& Chandraju, S. (2018). Z. Kristallogr. Cryst. Mater. 233, 349-360.
Macrae, C. F., Bruno, I. J., Chisholm, J. A., Edgington, P. R., McCabe, P., Pidcock, E., Rodriguez-Monge, L., Taylor, R., van de Streek, J. \& Wood, P. A. (2008). J. Appl. Cryst. 41, 466-470.

Prabhu, A. N., Jayarama, A., Subrahmanya Bhat, K., Manjunatha, K. B., Umesh, G. \& Upadhyaya, V. (2013). Indian J. Mater. Sci. 2013, 1-5.

Sheldrick, G. M. (2008). Acta Cryst. A64, 112-122.

Sheldrick, G. M. (2015). Acta Cryst. C71, 3-8.

Spackman, M. A. \& Jayatilaka, D. (2009). CrystEngComm, 11, 1932.

Spackman, M. A. \& McKinnon, J. J. (2002). CrystEngComm, 4, 378392.

Spackman, M. A., McKinnon, J. J. \& Jayatilaka, D. (2008). CrystEngComm, 10, 377-388.

Spek, A. L. (2009). Acta Cryst. D65, 148-155.

Turner, M. J., McKinnon, J. J., Wolff, S. K., Grimwood, D. J., Spackman, P. R., Jayatilaka, D. \& Spackman, M. A. (2017). CrystalExplorer17. University of Western Australia.

Westrip, S. P. (2010). J. Appl. Cryst. 43, 920-925. 


\section{supporting information}

Acta Cryst. (2019). E75, 53-57 [https://doi.org/10.1107/S2056989018017450]

Crystal structure and Hirshfeld surface analysis of a chalcone derivative: (E)-3-(4-fluorophenyl)-1-(4-nitrophenyl)prop-2-en-1-one

Qin Ai Wong, Tze Shyang Chia, Huey Chong Kwong, C. S. Chidan Kumar, Ching Kheng Quah and Md. Azharul Arafath

Computing details

Data collection: APEX2 (Bruker, 2009); cell refinement: SAINT (Bruker, 2009); data reduction: SAINT (Bruker, 2009); program(s) used to solve structure: SHELXS2013 (Sheldrick, 2008); program(s) used to refine structure: SHELXL2014 (Sheldrick, 2015); molecular graphics: SHELXTL (Sheldrick, 2008) and Mercury (Macrae et al., 2008); software used to prepare material for publication: PLATON (Spek, 2009) and publCIF (Westrip, 2010).

(E)-3-(4-Fluorophenyl)-1-(4-nitrophenyl)prop-2-en-1-one

Crystal data

$\mathrm{C}_{15} \mathrm{H}_{10} \mathrm{FNO}_{3}$

$M_{r}=271.24$

Monoclinic, $P 2_{1} / c$

$a=3.8860(5) \AA$

$b=13.2324(16) \AA$

$c=24.199(3) \AA$

$\beta=91.963(2)^{\circ}$

$V=1243.6(3) \AA^{3}$

$Z=4$

$F(000)=560$

$D_{\mathrm{x}}=1.449 \mathrm{Mg} \mathrm{m}^{-3}$

Mo $K \alpha$ radiation, $\lambda=0.71073 \AA$

Cell parameters from 4607 reflections

$\theta=2.3-30.4^{\circ}$

$\mu=0.11 \mathrm{~mm}^{-1}$

$T=296 \mathrm{~K}$

Block, yellow

$0.49 \times 0.35 \times 0.31 \mathrm{~mm}$

\section{Data collection}

Bruker SMART APEXII DUO CCD areadetector diffractometer

Radiation source: fine-focus sealed tube Graphite monochromator $\varphi$ and $\omega$ scans

Absorption correction: multi-scan (SADABS; Bruker, 2009)

$T_{\min }=0.794, T_{\max }=0.926$

10823 measured reflections

2418 independent reflections 1922 reflections with $I>2 \sigma(I)$

$R_{\text {int }}=0.026$

$\theta_{\text {max }}=26.0^{\circ}, \theta_{\min }=1.8^{\circ}$

$h=-4 \rightarrow 4$

$k=-16 \rightarrow 15$

$l=-29 \rightarrow 29$

Refinement

Refinement on $F^{2}$

Least-squares matrix: full

$R\left[F^{2}>2 \sigma\left(F^{2}\right)\right]=0.043$

$w R\left(F^{2}\right)=0.138$

$S=1.04$

2418 reflections

181 parameters

0 restraints

Hydrogen site location: inferred from neighbouring sites

$\mathrm{H}$-atom parameters constrained 
$w=1 /\left[\sigma^{2}\left(F_{\mathrm{o}}^{2}\right)+(0.072 P)^{2}+0.3042 P\right]$

where $P=\left(F_{\mathrm{o}}^{2}+2 F_{\mathrm{c}}^{2}\right) / 3$

$(\Delta / \sigma)_{\max }=0.001$

$$
\Delta \rho_{\max }=0.21 \mathrm{e} \AA^{-3}
$$

\section{Special details}

Geometry. All esds (except the esd in the dihedral angle between two 1.s. planes) are estimated using the full covariance matrix. The cell esds are taken into account individually in the estimation of esds in distances, angles and torsion angles; correlations between esds in cell parameters are only used when they are defined by crystal symmetry. An approximate (isotropic) treatment of cell esds is used for estimating esds involving 1.s. planes.

Fractional atomic coordinates and isotropic or equivalent isotropic displacement parameters $\left(\AA^{2}\right)$

\begin{tabular}{|c|c|c|c|c|}
\hline & $x$ & $y$ & $z$ & $U_{\text {iso }} * / U_{\text {eq }}$ \\
\hline $\mathrm{F} 1$ & $0.7362(4)$ & $-0.01394(9)$ & $0.57883(5)$ & $0.0899(5)$ \\
\hline $\mathrm{O} 1$ & $-0.1032(4)$ & $0.85915(12)$ & $0.77829(6)$ & $0.0784(5)$ \\
\hline $\mathrm{O} 2$ & $0.0898(6)$ & $0.97163(12)$ & $0.72386(8)$ & $0.1026(7)$ \\
\hline $\mathrm{O} 3$ & 0.7296 & $0.61266(9)$ & $0.55381(5)$ & $0.0665(4)$ \\
\hline N1 & $0.0476(4)$ & $0.88363(12)$ & $0.73723(6)$ & 0.0578 \\
\hline $\mathrm{C} 1$ & $0.3037(4)$ & $0.63109(12)$ & $0.68456(6)$ & $0.0460(4)$ \\
\hline $\mathrm{H} 1 \mathrm{~A}$ & 0.2995 & 0.5638 & 0.6955 & $0.055^{*}$ \\
\hline $\mathrm{C} 2$ & $0.1758(4)$ & $0.70499(13)$ & $0.71878(6)$ & $0.0479(4)$ \\
\hline $\mathrm{H} 2 \mathrm{~A}$ & 0.0857 & 0.6880 & 0.7527 & $0.057 *$ \\
\hline $\mathrm{C} 3$ & $0.1848(4)$ & $0.80391(12)$ & $0.70162(6)$ & $0.0443(4)$ \\
\hline $\mathrm{C} 4$ & $0.3144(5)$ & $0.83206(12)$ & $0.65149(7)$ & $0.0502(4)$ \\
\hline $\mathrm{H} 4 \mathrm{~A}$ & 0.3157 & 0.8995 & 0.6407 & $0.060 *$ \\
\hline $\mathrm{C} 5$ & $0.4418(4)$ & $0.75795(12)$ & $0.61790(6)$ & $0.0474(4)$ \\
\hline $\mathrm{H} 5 \mathrm{~A}$ & 0.5311 & 0.7757 & 0.5841 & $0.057 *$ \\
\hline C6 & $0.4388(4)$ & $0.65682(11)$ & $0.63385(6)$ & $0.0397(4)$ \\
\hline $\mathrm{C} 7$ & $0.5886(4)$ & $0.58109(12)$ & $0.59466(6)$ & $0.0437(4)$ \\
\hline $\mathrm{C} 8$ & $0.5671(4)$ & $0.47246(12)$ & $0.60636(7)$ & $0.0465(4)$ \\
\hline H8A & 0.4609 & 0.4501 & 0.6380 & $0.056^{*}$ \\
\hline $\mathrm{C} 9$ & $0.6995(4)$ & $0.40594(13)$ & $0.57171(6)$ & $0.0461(4)$ \\
\hline H9A & 0.8020 & 0.4332 & 0.5409 & $0.055^{*}$ \\
\hline $\mathrm{C} 10$ & $0.7052(4)$ & $0.29630(12)$ & $0.57557(6)$ & $0.0441(4)$ \\
\hline C11 & $0.8374(4)$ & $0.24150(13)$ & $0.53176(7)$ & 0.0498 \\
\hline H11A & 0.9211 & 0.2760 & 0.5015 & $0.060 *$ \\
\hline C12 & $0.8463(5)$ & $0.13763(14)$ & $0.53240(8)$ & $0.0573(5)$ \\
\hline $\mathrm{H} 12 \mathrm{~A}$ & 0.9314 & 0.1015 & 0.5029 & $0.069^{*}$ \\
\hline $\mathrm{C} 13$ & $0.7264(5)$ & $0.08894(13)$ & $0.57778(7)$ & $0.0571(5)$ \\
\hline $\mathrm{C} 14$ & $0.5966(5)$ & $0.13871(13)$ & $0.62234(7)$ & $0.0571(5)$ \\
\hline $\mathrm{H} 14 \mathrm{~A}$ & 0.5188 & 0.1032 & 0.6526 & $0.069^{*}$ \\
\hline $\mathrm{C} 15$ & $0.5851(4)$ & $0.24251(13)$ & $0.62076(7)$ & $0.0504(4)$ \\
\hline H15A & 0.4958 & 0.2776 & 0.6503 & $0.061 *$ \\
\hline
\end{tabular}

Atomic displacement parameters $\left(\AA^{2}\right)$

\begin{tabular}{lllllll}
\hline & $U^{11}$ & $U^{22}$ & $U^{33}$ & $U^{12}$ & $U^{13}$ & $U^{23}$ \\
\hline F1 & $0.1376(12)$ & $0.0406(6)$ & $0.0943(9)$ & $0.0055(6)$ & $0.0442(9)$ & $0.0029(6)$ \\
O1 & $0.1010(11)$ & $0.0756(10)$ & $0.0608(8)$ & $0.0045(8)$ & $0.0352(8)$ & $-0.0084(7)$
\end{tabular}




$\begin{array}{lllllll}\text { O2 } & 0.1626(18) & 0.0471(9) & 0.1018(12) & 0.0065(9) & 0.0579(12) & -0.0095(8) \\ \text { O3 } & 0.0962(10) & 0.0503(7) & 0.0550(7) & -0.0027(7) & 0.0350(7) & 0.0017(6) \\ \text { N1 } & 0.0665(10) & 0.0540(10) & 0.0534(9) & 0.0024(7) & 0.0107(7) & -0.0086(7) \\ \text { C1 } & 0.0552(10) & 0.0401(9) & 0.0430(8) & -0.0028(7) & 0.0065(7) & 0.0049(6) \\ \text { C2 } & 0.0546(9) & 0.0502(10) & 0.0395(8) & -0.0036(7) & 0.0103(7) & 0.0030(7) \\ \text { C3 } & 0.0465(9) & 0.0449(9) & 0.0417(8) & -0.0009(7) & 0.0050(7) & -0.0049(7) \\ \text { C4 } & 0.0622(10) & 0.0374(8) & 0.0515(9) & -0.0012(7) & 0.0107(8) & 0.0028(7) \\ \text { C5 } & 0.0580(10) & 0.0443(9) & 0.0406(8) & -0.0030(7) & 0.0110(7) & 0.0055(7) \\ \text { C6 } & 0.0409(8) & 0.0398(8) & 0.0386(8) & -0.0025(6) & 0.0027(6) & 0.0008(6) \\ \text { C7 } & 0.0469(9) & 0.0449(9) & 0.0397(8) & -0.0027(7) & 0.0056(6) & 0.0004(6) \\ \text { C8 } & 0.0502(9) & 0.0438(9) & 0.0459(8) & -0.0013(7) & 0.0098(7) & 0.0018(7) \\ \text { C9 } & 0.0475(9) & 0.0464(9) & 0.0447(8) & -0.0016(7) & 0.0061(7) & 0.0018(7) \\ \text { C10 } & 0.0424(8) & 0.0450(9) & 0.0450(8) & 0.0008(7) & 0.0056(7) & -0.0011(7) \\ \text { C11 } & 0.0562(10) & 0.0493(10) & 0.0449(9) & 0.0016(7) & 0.0155(7) & 0.0020(7) \\ \text { C12 } & 0.0689(11) & 0.0505(10) & 0.0538(10) & 0.0072(8) & 0.0206(9) & -0.0059(8) \\ \text { C13 } & 0.0689(12) & 0.0398(9) & 0.0634(11) & 0.0028(8) & 0.0151(9) & 0.0014(8) \\ \text { C14 } & 0.0711(12) & 0.0503(10) & 0.0512(10) & -0.0003(8) & 0.0194(8) & 0.0073(8) \\ \text { C15 } & 0.0586(10) & 0.0494(10) & 0.0443(9) & 0.0032(7) & 0.0147(7) & -0.0024(7) \\ & & & & & & \end{array}$

Geometric parameters $\left(\AA,{ }^{\circ}\right)$

\begin{tabular}{|c|c|c|c|}
\hline $\mathrm{F} 1-\mathrm{C} 13$ & $1.362(2)$ & $\mathrm{C} 7-\mathrm{C} 8$ & $1.468(2)$ \\
\hline $\mathrm{O} 1-\mathrm{N} 1$ & $1.2147(19)$ & $\mathrm{C} 8-\mathrm{C} 9$ & $1.331(2)$ \\
\hline $\mathrm{O} 2-\mathrm{N} 1$ & $1.221(2)$ & $\mathrm{C} 8-\mathrm{H} 8 \mathrm{~A}$ & 0.9300 \\
\hline $\mathrm{O} 3-\mathrm{C} 7$ & $1.2204(19)$ & $\mathrm{C} 9-\mathrm{C} 10$ & $1.454(2)$ \\
\hline $\mathrm{N} 1-\mathrm{C} 3$ & $1.473(2)$ & C9-H9A & 0.9300 \\
\hline $\mathrm{C} 1-\mathrm{C} 2$ & $1.385(2)$ & $\mathrm{C} 10-\mathrm{C} 11$ & $1.397(2)$ \\
\hline $\mathrm{C} 1-\mathrm{C} 6$ & $1.393(2)$ & $\mathrm{C} 10-\mathrm{C} 15$ & $1.398(2)$ \\
\hline $\mathrm{C} 1-\mathrm{H} 1 \mathrm{~A}$ & 0.9300 & $\mathrm{C} 11-\mathrm{C} 12$ & $1.375(2)$ \\
\hline $\mathrm{C} 2-\mathrm{C} 3$ & $1.374(2)$ & $\mathrm{C} 11-\mathrm{H} 11 \mathrm{~A}$ & 0.9300 \\
\hline $\mathrm{C} 2-\mathrm{H} 2 \mathrm{~A}$ & 0.9300 & $\mathrm{C} 12-\mathrm{C} 13$ & $1.369(3)$ \\
\hline $\mathrm{C} 3-\mathrm{C} 4$ & $1.381(2)$ & $\mathrm{C} 12-\mathrm{H} 12 \mathrm{~A}$ & 0.9300 \\
\hline $\mathrm{C} 4-\mathrm{C} 5$ & $1.377(2)$ & $\mathrm{C} 13-\mathrm{C} 14$ & $1.374(2)$ \\
\hline $\mathrm{C} 4-\mathrm{H} 4 \mathrm{~A}$ & 0.9300 & $\mathrm{C} 14-\mathrm{C} 15$ & $1.375(2)$ \\
\hline $\mathrm{C} 5-\mathrm{C} 6$ & $1.393(2)$ & $\mathrm{C} 14-\mathrm{H} 14 \mathrm{~A}$ & 0.9300 \\
\hline $\mathrm{C} 5-\mathrm{H} 5 \mathrm{~A}$ & 0.9300 & $\mathrm{C} 15-\mathrm{H} 15 \mathrm{~A}$ & 0.9300 \\
\hline $\mathrm{C} 6-\mathrm{C} 7$ & $1.510(2)$ & & \\
\hline $\mathrm{O} 1-\mathrm{N} 1-\mathrm{O} 2$ & $122.95(16)$ & $\mathrm{C} 9-\mathrm{C} 8-\mathrm{C} 7$ & $120.02(14)$ \\
\hline $\mathrm{O} 1-\mathrm{N} 1-\mathrm{C} 3$ & $118.80(16)$ & $\mathrm{C} 9-\mathrm{C} 8-\mathrm{H} 8 \mathrm{~A}$ & 120.0 \\
\hline $\mathrm{O} 2-\mathrm{N} 1-\mathrm{C} 3$ & $118.24(15)$ & $\mathrm{C} 7-\mathrm{C} 8-\mathrm{H} 8 \mathrm{~A}$ & 120.0 \\
\hline $\mathrm{C} 2-\mathrm{C} 1-\mathrm{C} 6$ & $120.53(14)$ & $\mathrm{C} 8-\mathrm{C} 9-\mathrm{C} 10$ & $128.67(15)$ \\
\hline $\mathrm{C} 2-\mathrm{C} 1-\mathrm{H} 1 \mathrm{~A}$ & 119.7 & $\mathrm{C} 8-\mathrm{C} 9-\mathrm{H} 9 \mathrm{~A}$ & 115.7 \\
\hline $\mathrm{C} 6-\mathrm{C} 1-\mathrm{H} 1 \mathrm{~A}$ & 119.7 & $\mathrm{C} 10-\mathrm{C} 9-\mathrm{H} 9 \mathrm{~A}$ & 115.7 \\
\hline $\mathrm{C} 3-\mathrm{C} 2-\mathrm{C} 1$ & $118.60(14)$ & $\mathrm{C} 11-\mathrm{C} 10-\mathrm{C} 15$ & $118.08(15)$ \\
\hline $\mathrm{C} 3-\mathrm{C} 2-\mathrm{H} 2 \mathrm{~A}$ & 120.7 & $\mathrm{C} 11-\mathrm{C} 10-\mathrm{C} 9$ & $118.33(14)$ \\
\hline $\mathrm{C} 1-\mathrm{C} 2-\mathrm{H} 2 \mathrm{~A}$ & 120.7 & $\mathrm{C} 15-\mathrm{C} 10-\mathrm{C} 9$ & $123.59(14)$ \\
\hline $\mathrm{C} 2-\mathrm{C} 3-\mathrm{C} 4$ & 122.43 & $\mathrm{C} 12-\mathrm{C} 11-\mathrm{C} 10$ & $121.34(15)$ \\
\hline
\end{tabular}




\begin{tabular}{|c|c|c|c|}
\hline $\mathrm{C} 2-\mathrm{C} 3-\mathrm{N} 1$ & $119.47(14)$ & $\mathrm{C} 12-\mathrm{C} 11-\mathrm{H} 11 \mathrm{~A}$ & 119.3 \\
\hline $\mathrm{C} 4-\mathrm{C} 3-\mathrm{N} 1$ & $118.09(15)$ & $\mathrm{C} 10-\mathrm{C} 11-\mathrm{H} 11 \mathrm{~A}$ & 119.3 \\
\hline $\mathrm{C} 5-\mathrm{C} 4-\mathrm{C} 3$ & $118.43(15)$ & $\mathrm{C} 13-\mathrm{C} 12-\mathrm{C} 11$ & $118.06(16)$ \\
\hline $\mathrm{C} 5-\mathrm{C} 4-\mathrm{H} 4 \mathrm{~A}$ & 120.8 & $\mathrm{C} 13-\mathrm{C} 12-\mathrm{H} 12 \mathrm{~A}$ & 121.0 \\
\hline $\mathrm{C} 3-\mathrm{C} 4-\mathrm{H} 4 \mathrm{~A}$ & 120.8 & $\mathrm{C} 11-\mathrm{C} 12-\mathrm{H} 12 \mathrm{~A}$ & 121.0 \\
\hline $\mathrm{C} 4-\mathrm{C} 5-\mathrm{C} 6$ & $120.95(14)$ & $\mathrm{F} 1-\mathrm{C} 13-\mathrm{C} 12$ & $118.38(16)$ \\
\hline $\mathrm{C} 4-\mathrm{C} 5-\mathrm{H} 5 \mathrm{~A}$ & 119.5 & $\mathrm{~F} 1-\mathrm{C} 13-\mathrm{C} 14$ & $118.37(16)$ \\
\hline $\mathrm{C} 6-\mathrm{C} 5-\mathrm{H} 5 \mathrm{~A}$ & 119.5 & $\mathrm{C} 12-\mathrm{C} 13-\mathrm{C} 14$ & $123.25(17)$ \\
\hline $\mathrm{C} 5-\mathrm{C} 6-\mathrm{C} 1$ & $119.06(14)$ & $\mathrm{C} 13-\mathrm{C} 14-\mathrm{C} 15$ & $118.00(15)$ \\
\hline $\mathrm{C} 5-\mathrm{C} 6-\mathrm{C} 7$ & $117.17(13)$ & $\mathrm{C} 13-\mathrm{C} 14-\mathrm{H} 14 \mathrm{~A}$ & 121.0 \\
\hline $\mathrm{C} 1-\mathrm{C} 6-\mathrm{C} 7$ & $123.78(14)$ & $\mathrm{C} 15-\mathrm{C} 14-\mathrm{H} 14 \mathrm{~A}$ & 121.0 \\
\hline $\mathrm{O} 3-\mathrm{C} 7-\mathrm{C} 8$ & $121.43(15)$ & $\mathrm{C} 14-\mathrm{C} 15-\mathrm{C} 10$ & $121.26(15)$ \\
\hline $\mathrm{O} 3-\mathrm{C} 7-\mathrm{C} 6$ & $118.36(14)$ & $\mathrm{C} 14-\mathrm{C} 15-\mathrm{H} 15 \mathrm{~A}$ & 119.4 \\
\hline $\mathrm{C} 8-\mathrm{C} 7-\mathrm{C} 6$ & $120.20(13)$ & $\mathrm{C} 10-\mathrm{C} 15-\mathrm{H} 15 \mathrm{~A}$ & 119.4 \\
\hline $\mathrm{C} 6-\mathrm{C} 1-\mathrm{C} 2-\mathrm{C} 3$ & $0.0(3)$ & $\mathrm{C} 1-\mathrm{C} 6-\mathrm{C} 7-\mathrm{C} 8$ & $6.1(2)$ \\
\hline $\mathrm{C} 1-\mathrm{C} 2-\mathrm{C} 3-\mathrm{C} 4$ & $0.4(3)$ & $\mathrm{O} 3-\mathrm{C} 7-\mathrm{C} 8-\mathrm{C} 9$ & $-0.8(3)$ \\
\hline $\mathrm{C} 1-\mathrm{C} 2-\mathrm{C} 3-\mathrm{N} 1$ & $179.54(15)$ & $\mathrm{C} 6-\mathrm{C} 7-\mathrm{C} 8-\mathrm{C} 9$ & $-179.99(15)$ \\
\hline $\mathrm{O} 1-\mathrm{N} 1-\mathrm{C} 3-\mathrm{C} 2$ & $-7.6(2)$ & $\mathrm{C} 7-\mathrm{C} 8-\mathrm{C} 9-\mathrm{C} 10$ & $-179.96(15)$ \\
\hline $\mathrm{O} 2-\mathrm{N} 1-\mathrm{C} 3-\mathrm{C} 2$ & $172.67(18)$ & $\mathrm{C} 8-\mathrm{C} 9-\mathrm{C} 10-\mathrm{C} 11$ & $175.31(17)$ \\
\hline $\mathrm{O} 1-\mathrm{N} 1-\mathrm{C} 3-\mathrm{C} 4$ & $171.54(17)$ & $\mathrm{C} 8-\mathrm{C} 9-\mathrm{C} 10-\mathrm{C} 15$ & $-4.6(3)$ \\
\hline $\mathrm{O} 2-\mathrm{N} 1-\mathrm{C} 3-\mathrm{C} 4$ & $-8.2(3)$ & $\mathrm{C} 15-\mathrm{C} 10-\mathrm{C} 11-\mathrm{C} 12$ & $0.8(3)$ \\
\hline $\mathrm{C} 2-\mathrm{C} 3-\mathrm{C} 4-\mathrm{C} 5$ & $-0.6(3)$ & $\mathrm{C} 9-\mathrm{C} 10-\mathrm{C} 11-\mathrm{C} 12$ & $-179.15(16)$ \\
\hline $\mathrm{N} 1-\mathrm{C} 3-\mathrm{C} 4-\mathrm{C} 5$ & $-179.74(15)$ & $\mathrm{C} 10-\mathrm{C} 11-\mathrm{C} 12-\mathrm{C} 13$ & $-1.0(3)$ \\
\hline $\mathrm{C} 3-\mathrm{C} 4-\mathrm{C} 5-\mathrm{C} 6$ & $0.3(3)$ & $\mathrm{C} 11-\mathrm{C} 12-\mathrm{C} 13-\mathrm{F} 1$ & $-179.66(17)$ \\
\hline $\mathrm{C} 4-\mathrm{C} 5-\mathrm{C} 6-\mathrm{C} 1$ & $0.1(3)$ & $\mathrm{C} 11-\mathrm{C} 12-\mathrm{C} 13-\mathrm{C} 14$ & $0.4(3)$ \\
\hline $\mathrm{C} 4-\mathrm{C} 5-\mathrm{C} 6-\mathrm{C} 7$ & $-179.16(15)$ & $\mathrm{F} 1-\mathrm{C} 13-\mathrm{C} 14-\mathrm{C} 15$ & $-179.50(17)$ \\
\hline $\mathrm{C} 2-\mathrm{C} 1-\mathrm{C} 6-\mathrm{C} 5$ & $-0.3(2)$ & $\mathrm{C} 12-\mathrm{C} 13-\mathrm{C} 14-\mathrm{C} 15$ & $0.5(3)$ \\
\hline $\mathrm{C} 2-\mathrm{C} 1-\mathrm{C} 6-\mathrm{C} 7$ & $178.91(15)$ & $\mathrm{C} 13-\mathrm{C} 14-\mathrm{C} 15-\mathrm{C} 10$ & $-0.7(3)$ \\
\hline $\mathrm{C} 5-\mathrm{C} 6-\mathrm{C} 7-\mathrm{O} 3$ & $6.1(2)$ & $\mathrm{C} 11-\mathrm{C} 10-\mathrm{C} 15-\mathrm{C} 14$ & $0.1(3)$ \\
\hline $\mathrm{C} 1-\mathrm{C} 6-\mathrm{C} 7-\mathrm{O} 3$ & $-173.10(16)$ & $\mathrm{C} 9-\mathrm{C} 10-\mathrm{C} 15-\mathrm{C} 14$ & $-179.98(16)$ \\
\hline $\mathrm{C} 5-\mathrm{C} 6-\mathrm{C} 7-\mathrm{C} 8$ & $-174.65(15)$ & & \\
\hline
\end{tabular}

Hydrogen-bond geometry $\left(A,{ }^{\circ}\right)$

\begin{tabular}{lllll}
\hline$D-\mathrm{H} \cdots A$ & $D-\mathrm{H}$ & $\mathrm{H} \cdots A$ & $D \cdots A$ & $D-\mathrm{H}^{\cdots} A$ \\
\hline $\mathrm{C} 4-\mathrm{H} 4 A \cdots \mathrm{F} 1^{\mathrm{i}}$ & 0.93 & 2.53 & $3.183(2)$ & 128 \\
$\mathrm{C} 11-\mathrm{H} 11 A \cdots \mathrm{O} 3^{\mathrm{ii}}$ & 0.93 & 2.43 & $3.329(2)$ & 161 \\
$\mathrm{C} 15-\mathrm{H} 15 A \cdots \mathrm{O} 1^{\mathrm{iii}}$ & 0.93 & 2.58 & $3.489(2)$ & 166 \\
\hline
\end{tabular}

Symmetry codes: (i) $x, y+1, z$; (ii) $-x+2,-y+1,-z+1$; (iii) $-x, y-1 / 2,-z+3 / 2$. 